\title{
Social communication disorder: a narrative review on current insights
}

This article was published in the following Dove Press journal:

Neuropsychiatric Disease and Treatment

\author{
Zehra Topal' \\ Nuran Demir Samurcu ${ }^{2}$ \\ Sarper Taskiran ${ }^{3}$ \\ Ali Evren Tufan ${ }^{4}$ \\ Bengi Semerci $i^{5}$ \\ 'Department of Child and Adolescent \\ Psychiatry, Hakkari State Hospital, \\ Hakkari, Turkey; ${ }^{2}$ Department of Child \\ and Adolescent Psychiatry, Izzet Baysal \\ Hospital for Maternity and Pediatric \\ Disorder, Bolu, Turkey; ${ }^{3}$ Department \\ of Psychiatry, Koc University Medical \\ Faculty, Istanbul, Turkey; ${ }^{4}$ Department \\ of Child and Adolescent Psychiatry, \\ Abant Izzet Baysal University Medical \\ Faculty, Bolu, Turkey; ${ }^{5}$ Department of \\ Psychology, Bengi Semerci Institute, \\ Hasan Kalyoncu University, Istanbul, \\ Turkey
}

\begin{abstract}
Social communication disorder (SCD) is a novel diagnosis listed under the rubric of communication disorders within the Diagnostic and Statistical Manual of Mental Disorders-5 (DSM-5) and it is reported to be characterized by impairment in use of verbal and nonverbal communication for social aims. This review attempts to summarize the current understanding of the SCD concept along with its evolution and presents data from previous studies conducted. Suggestions for further research are also delineated. As listed in DSM-5, the criteria for this novel diagnosis are vague, display elevated comorbidity with other neurodevelopmental disorders and other childhood psychopathologies, and show partial overlap with autistic spectrum disorders both in terms of genetics and family histories. Data on cross-cultural presentations and temporal stability are also limited. The social communication model proposed by Catani and Bambini may help integrate the neurobiological findings pertaining to SCD. Valid and reliable assessment methods need to be developed for SCD. This may involve either development of novel instruments capturing the DSM-5 criteria or application of statistical methods such as item response theory to existing instruments. The relationships between broad autism phenotype, pragmatic language impairment, nonverbal learning disorder, learning disorders, autistic spectrum disorders, and SCD should be evaluated with further studies.
\end{abstract}

Keywords: social communication disorder, pragmatics, autistic spectrum disorder, neurodevelopment

\section{Introduction}

Social communication disorder (SCD) is a novel diagnosis listed under the rubric of communication disorders within the Diagnostic and Statistical Manual of Mental Disorders-5 (DSM-5) and it is reported to be characterized by impairment in use of verbal and nonverbal communication for social aims. As listed in DSM-5, those with the diagnosis are impaired in processing implied sentences and indirect uses of language such as metaphors, humor, and aphorisms. They also display nonverbal communication problems along with verbal ones, such as greeting others according to context, waiting for turns in conversations, and modulating their behaviors according to context. According to DSM-5 criteria, SCD can be diagnosed along with other communication disorders while the presence of autistic spectrum disorder (ASD) is a criterion for exclusion. ${ }^{1}$

In this narrative review of relevant diagnoses from the existing literature, we aimed to summarize what is known about the background of SCD diagnosis, the reasons for its introduction into DSM-5, its clinical presentation, and problems about diagnosis/ differential diagnosis, culminating in the current knowledge base about its biological basis and treatment.
Correspondence: Ali Evren Tufan Department of Child and Adolescent Psychiatry, Abant Izzet Baysal University Medical Faculty, Golkoy, 14280,

Bolu, Turkey

Tel +905325269416

Email tevrenus@yahoo.com 


\section{History and sources of SCD concept}

Children impaired in social uses of language have been recognized for a long time within the speech therapy literature. ${ }^{2}$ However, terms used differed among authors and study centers with such children reported as displaying "semantic-pragmatic syndrome", 3,4 "semantic-pragmatic difficulties", 5 "conversational disability", 6 "pragmatic disability", "semantic-pragmatic disorder", ${ }^{8}$ and last and most recently "pragmatic language impairment (PLI)"., 9,10

Traditionally, those children with PLI were defined as displaying inappropriate/ineffective use of language and gestures in social contexts ${ }^{11}$ and initial conceptualizations underlined the presence of social relationship problems distinctive from those seen in autistic disorders. ${ }^{12-14}$ According to those studies, structural components of language (eg, vocabulary and grammar) in children with PLI were relatively preserved, while context-dependent use and understanding of language and following social rules/conventions of language use were impaired..$^{10}$ The majority of those children were reported not to display repetitive, restrictive behaviors and interests more typical of children with ASDs. ${ }^{10}$ Problems of social communication including pragmatics are also listed among the core symptoms of ASDs. Therefore, similarities and differences between children with PLI in speech/language disorders literature and those with high-functioning autism/Asperger syndrome/pervasive developmental disorder not otherwise specified (PDD-NOS) in child psychiatric literature have been much underlined, and convergent/divergent validities of those concepts have been discussed. ${ }^{11,15}$

Autism which is the prototype of ASDs was first described by Kanner as involving core symptoms of limited social interaction and communication as well as restricted and repetitive behavioral patterns. Successive diagnostic systems have combined severity and presence of problems in those domains in variable combinations to characterize ASDs. ${ }^{16}$ However, the exact nature of language problems in ASD was difficult to delineate due to extreme variability. ${ }^{17,18}$ To better understand the sources of variability, the DSM$I V-T R$ criteria for autistic disorder (299.0 DSM-IV-TR) may be reviewed. ${ }^{19}$ According to $D S M-I V-T R$, for a diagnosis of autistic disorder, at least two symptoms of qualitative impairment in social interaction should be present out of a total of four (ie, 16 different combinations) along with at least one each from four criteria of qualitative impairments in communication (ie, four different combinations) and four criteria from restricted repetitive and stereotyped patterns of behavior, interests, and activities (ie, a further four different combinations). With those criteria, a total of $16 \times 4 \times 4=256$ combinations are possible. ${ }^{20}$ When 6 out of 12 criteria from three domains are required with replacement, a total of 2,985.984 different presentations of autistic disorder is possible, at least in theory. With those caveats in mind, the consensus was that $\sim 50.0 \%$ of children with ASD did not have functional language, and Jarrold et $\mathrm{al}^{17}$ reported that language in patients with ASDs was characterized with better development of articulation compared with other areas, superior verbal expression (vs verbal comprehension), and lexical comprehension (vs grammatical comprehension). Paul et al reported that children with ASDs had problems in grammar and pragmatics that include use of combined words, functional words, and markers, whereas Eigsti et al reported that they had delayed development of syntax and performed inconsistently in terms of pragmatics. ${ }^{21,22}$ Accordingly, the general literature points to grammatical, lexical, and pragmatic deficits in children with ASDs, although the relationships between those aspects and their magnitudes relative to each other were not clear. ${ }^{18}$

Neuropsychologists also describe a group of children similar to those with PLI within the speech/language disorders literature in alternative terms (eg, nonverbal learning disabilities [NVLD] ). ${ }^{23,24}$ The symptoms of NVLD were listed as problems with visual spatial processing and visual motor coordination, dysfunctional tactile perception, attentional difficulties, limitations in abstract reasoning, problem solving, emotional perception, and social communication. The original description also added that the syndrome was especially seen among 9 - to 15 -year-olds and that rote verbal skills were better than comprehension. ${ }^{23,24}$

As can be seen, PLI, ASDs, and NVLD all reflected problems in social communication in varying degrees. But a point that is not usually touched upon by researchers focusing on PLI/ASDs/NVLD is that higher-order language skills (ie, inference, comparison, and decoding) may also be impaired in children with specific language impairment (SLI) and that children with expressive or mixed receptive/ expressive language disorders may also have problems in organizing/expressing thoughts both in speaking and in writing, although nonverbal communication skills are preserved..$^{18,24}$ When those observations were considered, some authors suggested that SLI and ASDs may lie on a continuum of social-pragmatic communication problems with PLI situated in between. ${ }^{18,25}$ Some studies suggest this view ${ }^{26}$ while this approach was also criticized as being somewhat reductionist. ${ }^{18}$

Although categorical approaches to childhood psychopathology have proven useful, it is not easy to "carve the 
nature at its joints", especially for social-pragmatic disorders. Accordingly, researchers have reported that children with attention-deficit hyperactivity disorder (ADHD), learning disorders (LDs), and even those with obsessive compulsive disorder (OCD) also have problems in social communication and pragmatics. ${ }^{26-28}$ This position is contended by some of the researchers, however, who maintain that problems in social communication and pragmatics may not be specific to childhood psychopathologies and that comorbidity with disruptive behavior disorders and/or NVLD, age, and social experience all play important roles in determining the severity of those problems..$^{29,30}$ Recognizing the problems inherent in overinclusivity and lack of diagnostic validity, some authors proposed that pragmatic difficulties may be better conceptualized as a dimensional symptom displayed across a range of neurodevelopmental disorders. ${ }^{31}$

As for the current paradigm, important revisions in diagnosing children with social difficulties have been made with the latest edition of DSM-5. DSM-5 combines the categories of communication and social interaction in the single domain of 'social communication problems' to reduce heterogeneity in clinical presentation and defines another disorder (ie, $\mathrm{SCD}$ ) related with those problems. The ASDs in DSM-5 are characterized with social communication problems and restrictive/repetitive behaviors (RRBs) and interests and the SCD is characterized by isolated problems in social communication. ${ }^{1}$ However, there also exist some studies suggesting that RRB may also be observed in milder forms among children with PLI/SCD and that those disorders may best be conceptualized as a continuum. ${ }^{32}$

\section{Reasons for including SCD diagnosis under DSM-5}

Until recent years, the behavioral symptoms of autism were thought to have a common genetic, neurological, and cognitive basis. Earlier studies have demonstrated that social impairments and repetitive-sterotypical behavioral and language problems correlated significantly and, therefore, should be clustered together. ${ }^{33,34}$ However, more recent studies showed that symptoms pertaining to social communication and RRBs did not correlate as highly as expected and that samples with clinically significant symptoms pertaining to a domain may not display symptoms from the other domain at a clinically significant level. ${ }^{35}$ Furthermore, some of the studies reported that some of the subjects displayed only one of the symptom clusters such as social communication (SC) and/or restrictive/repetitive behaviors (RRB). ${ }^{11,18,36}$ Those results supported a distinction between a group of children displaying prominent RRBs and another group displaying social communication problems. The group with RRB symptoms was thought to belong to ASD, whereas the latter group was thought to be separate.

ASDs were classified under the heading of PDD in $D S M-I V-T R$ and subclassifications were listed as autistic disorder, Asperger's disorder, PDD-NOS, Rett disorder, and childhood disintigrative disorder. ${ }^{1,19}$ Rett disorder and childhood disintigrative disorder were removed from this group in DSM-5 due to their genetic and neurological bases. ${ }^{1}$ As for the former three, an influential study showed that autistic disorder, Asperger's disorder, and PDD-NOS were similar to each other and the only predictors of receiving one of those diagnoses in place of others were the center at which the evaluation was conducted along with the severity of symptoms displayed. ${ }^{37}$ This finding eased the way of accepting ASD as a term and abolishing subdiagnoses such as Asperger's syndrome and PDD-NOS, although there were criticisms against such a move. ${ }^{16}$ The main criticisms involved the effects of changes on toddlers/infants who may have no/subtle RRB symptoms and those who received PDD-NOS diagnosis as per DSM-IV-TR and were helped by interventions. ${ }^{16}$ DSM-5 addressed those concerns by adding the proviso that children who received PDD-NOS diagnosis will receive the ASD diagnosis regardless of criteria. ${ }^{1}$

There was also the problem of rising rates of autistic disorder. The prevalence of autistic disorder in DSM-IV was reported to be $4-5: 10.000 .{ }^{38}$ The rate for ASDs in the USA for the year 2010 was 1:68, whereas for 2011-2012, it was $2.0 \%{ }^{39,40}$ A community-based epidemiological study conducted in South Korea found the prevalence for DSM-IV-TR PDD as $2.64 \%$, whereas the rate for ASD as per DSM-5 was $2.20 \%$. The authors reported that the difference between rates could be explained by cases who met the criteria for SCD as per DSM-5 and that their findings supported rising rates of ASDs in countries other than the US. ${ }^{41}$ Those findings supported the view that milder cases who were being diagnosed with PDD-NOS may be responsible for the rise in prevalence and that addition of a novel category for isolated problems in social-pragmatic communication may both reduce the inflation of prevalence and provide a more homogenous sample of patients for further studies. ${ }^{37,41}$ On the other hand, genetically informed community epidemiological studies showed that genetic etiology of social communication problems partially overlap with those for ASDs and that the quest for genetic homogeneity among patient samples may be harder than previously thought. ${ }^{42}$

Those results, despite their limited nature, eased the evolution of the PDD category in DSM- $I V$ to ASD in DSM-5 
along with the introduction of SCD diagnosis. The category of PDD-NOS listed under PDD in DSM- $I V$ denoted atypical clinical presentations with an incomplete presentation of autism but required clinical support and services. Such children who did not display full-blown autism could receive necessary clinical and educational services by virtue of this label. The PDD-NOS diagnosis described patients with problems in reciprocal social interaction or had symptoms of RRB but did not fit with other diagnoses listed under the rubric of PDD. Because symptoms of RRB are sine qua non for ASD diagnosis under DSM-5, there were suggestions that a subgroup of patients with PDD-NOS (ie, those with social communication problems but without RRB symptoms) may be left without required medical and educational services. ${ }^{1,16,43}$ Although as reported, the DSM-5 addressed those concerns.

\section{Clinical presentation of SCD and problems in diagnosis}

SCD as listed in DSM-5 is listed under the rubric of communication disorders and is characterized by the presence of chronic problems in social use of verbal and nonverbal communication. The symptoms and findings described in DSM-5 include impairments in social use of communication and/or obeying social rules and conventions of language (ie, greeting in accordance with context and sharing information), impairments in changing communicative style in accordance with the situation or needs of the listening party, waiting for conversational turns, reformulating communicative intent in case of misunderstandings, knowing how to use verbal and nonverbal iconic gestures in context of speech, and problems in inferring the implied message with metaphors/ironies/ similes/aphorisms. The listed impairments and problems hinder effective communication, social participation, formation, and maintenance of social relationships and lead to academic/vocational/interpersonal dysfunction. ${ }^{1}$

Features supporting diagnosis as per DSM-5 include a delay in acquiring spoken language, along with current/ lifetime structural language disorders. The affected patients are also reported to display elevated levels of ADHD, LDs, and behavioral problems. ${ }^{1}$

Because social communication requires adequate development of speech and language beforehand, SCD may be considered as a diagnosis only after 4-5 years. ${ }^{1,43}$ DSM-5 reports that although symptoms may start in early development, milder forms may not be detected until early adolescence, and impairment may become apparent with demands of social interaction, which increase and become more complicated by adolescence and overcome the already limited capacity of the child. ${ }^{1}$

As listed in DSM-5, the SCD concept is vague, relationships to LDs, PLI, and ASDs are not specified, displays high comorbidity, and its temporal stability is not clear, leading to problems with diagnostic validity. ${ }^{31}$ There are also problems in delineating the concept from broad autism phenotype (BAP). ${ }^{44}$ The BAP construct involves language/communication problems, social interaction difficulties, repetitive/ restricted interests/behavior, and rigid/aloof personality traits observed in relatives of probands with ASDs. ${ }^{45}$ Also, although guides to DSM-5 mental status examinations involve sample interview questions for SCD (ie, introducing one's self and greetings) there are no widely used valid and reliable diagnostic instruments on par with Autism Diagnostic InterviewRevised or autism diagnostic observation schedule. ${ }^{46}$

Apart from the primary symptoms that characterize the disorder, SCD is also reported to present in clinical samples with secondary problems arising from the core deficits. Focusing on the impairments in social communication may preclude a global evaluation of the patient and lead to missing secondary problems and diagnoses. For example, problems in sense of agency, development of self, and emotion regulation may be frequently observed in children with SCD. ${ }^{47,48}$ "Sense of agency" denotes the ability to achieve personal autonomy, control and take responsibility for decisions pertaining to one's own life, cope with barriers in life, and progress toward selected personal goals. ${ }^{49}$ "Narrative skill" helps organize life events and experiences, and restructure them leading to a coherent personal narrative and shaping of personality. The impairments in narrative skills in patients with SCD may lead to impairments in personality and self-development. ${ }^{49}$ Similarly, for adaptive and efficient emotion regulation development of social interaction and communication skills along with recognition, expression and understanding of emotions both in one's own self and in others are needed. ${ }^{50}$ Therefore, patients with SCD probably also experience difficulties in emotion regulation. ${ }^{50}$

\section{Differential diagnosis of SCD Differential diagnosis with ASDs}

According to current diagnostic criteria, ASD diagnosis takes precedence in evaluating patients displaying problems of social communication. ${ }^{1}$ Both SCD and ASD are characterized by difficulties in social communication and interaction, whereas ASD diagnosis requires the presence of RRB symptoms during evaluation and/or past history of RRB symptoms if they are currently remitted. ${ }^{1}$ Therefore, an adequate diagnosis of 
SCD and its differentiation from ASDs requires a detailed developmental history, preferably from multiple sources in addition to a cross-sectional mental status examination. ${ }^{1,46}$

However, a deeper perusal of the DSM-5 criteria reveals contradictory information on the importance of RRB symptoms in differential diagnosis. According to DSM-5, a diagnosis of ASD requires at least two symptoms from the RRB domain (B criterion) either at the current evaluation or with life-time history. ${ }^{1} \mathrm{SCD}$ diagnosis on the other hand requires absence of RRB symptoms both at the current evaluation and with life-time history. ${ }^{1}$ Those criteria leave patients with subthreshold RRB symptoms (ie, $<2$ ) at the current evaluation and life-time history who display social communication difficulties in a diagnostic limbo. ${ }^{1,51}$

\section{Differential diagnosis with other communication disorders}

Other disorders listed under the rubric of communication disorders in DSM-5 include language disorder, speech sound disorder, and childhood onset fluency disorder (stuttering). ${ }^{1}$ Language disorder is characterized by problems in acquisition and use of language across modalities (ie, syntax, grammar, and lexicon) due to deficits in comprehension or production which lead to dysfunction. ${ }^{1}$ Speech sound disorder is characterized by problems in production of speech sounds which interfere with communication. ${ }^{1}$ Last, childhood onset speech fluency disorder is characterized by problems in fluency of speech which impacts functioning. ${ }^{1}$

All of those disorders may lead to secondary problems in social communication and complicate their differentiation from SCD. Anticipating this problem, the DSM-5 notes that SCD may be diagnosed in addition to other communication disorders. ${ }^{1}$ Regardless of this note, comorbidity of SCD with other communication disorders may be high and the threshold to denote a primary diagnosis of SCD versus secondary problems of social communication superimposed on other communication disorders may be arbitrary. ${ }^{51}$

\section{Differential diagnosis with ADHD}

ADHD is a neurodevelopmental disorder characterized by developmentally inappropriate and impairing symptoms of inattention, hyperactivity, and impulsivity. ${ }^{1}$ Those symptoms in turn may lead to difficulties in social interaction and communication. ${ }^{52}$ Inattention may lead to missed cues in communication while hyperactivity/impulsivity may lead to problems in waiting for conversational turns. Symptoms from all domains may cause problems in modulating communication style according to the other's needs. ${ }^{52}$
Further complicating this picture, SCD is reported to be frequently comorbid with ADHD. ${ }^{1}$ The differentiating features of social communication difficulties primarily due to ADHD symptoms and those primarily due to SCD, if any, are not clear. Logically, social communication difficulties primarily due to ADHD may respond to pharmacotherapy while those primarily due to SCD may not. As far as we are aware, there exist no studies currently testing this hypothesis.

Social anxiety disorder and intellectual disability may also affect social communication and be listed in differential diagnosis. For the former, anxiety in social situations and developmental history may be crucial, whereas for the latter, a global delay across all domains of development may point to the diagnosis. ${ }^{1}$

\section{Genetic basis for SCD}

As reported previously, genetic etiologies for SCD and ASDs may partially overlap. ${ }^{42}$ Regardless of this overlap, there are some interesting findings concerning SCD. Demily et al reported an adult with SCD without intellectual deficiency and duplication at 19p13.2p12 leading to suggestions that copy number variation may contribute to SCD. ${ }^{53}$ Campbell et al reported that MET promoter variant rs $1858830 \mathrm{C}$ allele of the receptor tyrosine kinase is related to social communication difficulties in 748 individuals with ASDs from 367 families. ${ }^{54}$ In probands with $\mathrm{OCD}$ and their relatives, elevated pragmatic rating scale scores were associated with $\mathrm{Chr} 12$ and $\mathrm{X}$, whereas those with lower scores were associated with $\mathrm{Chr} 3 .{ }^{28}$ And in a preclinical study, Sungur et al reported that SHANK1knockout mice vocalized less and that this communication deficit was more prominent in social contexts. ${ }^{55}$ Those results, although limited, may lead to interesting avenues for the genetic etiology of SCD. In the light of those findings, $3,7 q, 12,19$ p, 19q, and X chromosomes may lead to fruitful avenues of research on SCD.

\section{Neuroanatomy of SCD}

As with other diagnoses, isolated significant results in neuroanatomical studies of SCD are legion and the main limitation is their integration to a coherent whole. Accordingly, Qiu et al reported that deformations of the bilateral medial caudate head in boys with ASD correlated with greater social communication problems. ${ }^{56}$ Chien et al reported that the integrity of the right frontoparietal tracts was strongly correlated with the social communication subscores of the social communication questionnaire (SCQ) in adolescents and young adults with ASD. ${ }^{57}$ Lo et al found that in boys with ASDs, fractional anisotropy of the superior longitudinal 
fasciculus and frontal aslant tracts was related with social interaction subscale of SCQ. ${ }^{58}$ Mitchell et al found that social communication deficits in co-twins of monozygotic children with ASDs correlated with changes in cerebellar vermis. ${ }^{59}$

A recent model proposed by Catani and Bambini for a language network aimed at social communication may help with integration of those results. ${ }^{60}$ The network includes representation of informative actions, communicative intentions, lexica/semantic processing, analysis of syntax and integration of pragmatics in increasing order of complexity. According to Catani and Bambini, the superior longitudinal fasciculus forms the frontoparietal network for informative actions, frontal aslant tracts form the frontal aslant network for communicative intentions, uncinate, inferior longitudinal and inferior frontal-occipital fasciculi form the anterior temporal network for lexical/semantic processing, arcuate fasciculus forms the frontotemporal network for analysis of syntax, and isolated tracts in temporoparietal junction form the temporoparietal network for integration of pragmatics. Accordingly, it may be proposed that the social pragmatic communication deficits in SCD and in other disorders may arise from problems within this network, although further studies are necessary.

\section{Treatments for SCD}

There are isolated reports of cases with SCD who benefited from individualized therapy ${ }^{61}$ as well as effects of therapy targeted on social communication and play in preschoolers with ASDs. ${ }^{62}$ Data from animal models and early clinical trials suggest that novel and existing compounds, including $N$-methyl-D-aspartate modulators, gamma-aminobutyric acid agonists, metabotropic glutamate receptor antagonists, and neuropeptides, may enhance social communication/function in ASD. ${ }^{63}$ On the other hand, theory of mind as well as augmented/alternative communication interventions were shown to have no effect on social communication in ASD, although their effects - if any - on children with SCD remain to be seen. ${ }^{64,65}$

\section{Conclusion}

This review attempted to summarize the current insights pertaining to the SCD diagnosis introduced in DSM-5. Although children impaired in social-communicative aspects of language have been recognized for a long time within the speech/ language disorders literature, the introduction of SCD diagnosis placed those children firmly in the spotlight. As listed in DSM-5, the criteria for this novel diagnosis are vague, display elevated comorbidity with other neurodevelopmental disorders and other childhood psychopathologies, and show partial overlap with ASDs in terms of both genetics and family histories. ${ }^{1,18,31,43,66}$ Data on cross-cultural presentations and temporal stability are also limited. ${ }^{1,31}$ The social communication model proposed by Catani and Bambini may help integrate the neurobiological findings pertaining to SCD. ${ }^{60}$ Valid and reliable assessment methods need to be developed for SCD. This may involve either the development of novel instruments capturing the DSM-5 criteria or application of statistical methods such as item response theory to existing instruments. The relationships among BAP, PLI, NVLD, LDs, ASDs, and SCD should be evaluated with further studies.

\section{Acknowledgments}

Parts of this paper were presented in the panel "Social Communication Disorder or Asperger Syndrome" in the 24th National Congress of Child and Adolescent Mental Health and Disorders (2014, Konya, Turkey) and in the poster titled "DSM-5 diagnoses of children and adolescents diagnosed with Pervasive Developmental Disorders according to the $D S M-I V-T R$ system and predictors of social communication disorder diagnosis" in the 23rd National Congress of Child and Adolescent Mental Health and Disorders (2013, Edirne, Turkey). The paper has not been submitted before in its entirety for potential publication and is not under consideration for publication elsewhere.

\section{Author contributions}

All authors contributed toward data analysis, drafting and revising the paper and agree to be accountable for all aspects of the work.

\section{Disclosure}

The authors report no conflicts of interest in this work.

\section{References}

1. American Psychiatric Association. Diagnostic and Statistical Manual of Mental Disorders, DSM-5. 5th ed. Arlington: American Psychiatric Association; 2013:47-49.

2. Bara BG, Bosco FM, Bucciarelli M. Developmental pragmatics in normal and abnormal children. Brain Lang. 1999;68(3):507-528.

3. Rapin I, Allen DA. Developmental language disorders: nosologic considerations. In: Kirk U, editor. Neuropsychology of Language, Reading, and Spelling. New York: Academic; 1983:155-184.

4. Rapin I, Allen DA. The semantic-pragmatic deficit disorder: classification issues. Int J Lang Comm Disord. 1998;33(1):71-108.

5. Vance M, Wells B. The wrong end of the stick: language-impaired children's understanding of non-literal language. Child Lang Teach Ther. 1994; 10:23-46.

6. Conti-Ramsden G, Gunn M. The development of conversational ability: a case study. Br J Disord Comm. 1986;21:339-51.

7. McTear M, Conti-Ramsden G. Pragmatic Disability in Children. London: Whurr; 1992. 
8. Bishop DVM, Rosenbloom I. Classification of childhood language disorders. In: Yule W, Rutter M, editors. Clinics in Developmental Medicine, vol. 101/102. MacKeith: London; 1987:16-41.

9. Bishop DVM. Development of the children's communication checklist (CCC): a method for assessing qualitative aspects of communicative impairment in children. J Child Psychol Psychiatry. 1998;39(6): 879-893.

10. Botting N, Conti-Ramsden G. Pragmatic language impairment without autism: the children in question. Autism. 1999;3:371-396.

11. Russell RL. Social communication impairments: pragmatics. Pediatr Clin North Am. 2007;54(3):483-506.

12. Cromer RF. Developmental language disorders: cognitive processes, semantics, pragmatics, phonology, and syntax.JAutism Dev Disord. 1981; 11(1):57-74.

13. Prutting CA. Pragmatics as social competence. J Speech Hear Disord. 1982;47(2):123-134.

14. Prutting CA. The pragmatics of language: theoretical and applied issues. S Afr J Commun Disord. 1984;31:3-5.

15. Fernandes FDM, Amato CAH, Molini-Avejonas DR. Language assessment in Autism. In: Mohammadi M-R, editor. A Comprehensive Book on Autism Spectrum Disorders. Rijeka, Croatia: InTech; 2011:3-22.

16. Tsai LY, Ghaziuddin M. DSM-5 ASD moves forward into the past. J Autism Dev Disord. 2014;44:321-330.

17. Jarrold C, Boucher J, Russell J. Language profiles in children with autism: theoretical and methodological implications. Autism. 1997; 1:57-76.

18. Bishop DV. Autism and specific language impairment: categorical distinction or continuum. Novartis Found Symp. 2003;251:213-226.

19. American Psychiatric Association. Diagnostic and Statistical Manual of Mental Disorders-Fourth Edition-Text Revision (DSM-IV-TR). Washington, DC: American Psychiatric Association; 2000.

20. Combinations and permutations calculator. Available from http://stattrek.com/online-calculator/combinations-permutations.aspx. Accessed July 7, 2017.

21. Paul R, Miles S, Cicchetti D, et al. Adaptive behavior in autism and pervasive developmental disorder - not otherwise specified: microanalysis of scores on the Vineland Adaptive Behavior Scale. J Autism Dev Disord. 2004;34(2):223-228.

22. Eigsti IM, Benetto L, Dadlani M. Beyond pragmatics: morphosyntactic development in autism. J Autism Dev Disord. 2007;37: 1007-1023.

23. Rourke BP, Young GC, Leenaars AA. A childhood learning disability that predisposes those afflicted to adolescent and adult depression and suicide risk. J Learn Disabil. 1989;22(3):169-175.

24. Niskala Apps J, Newby RF, Roberts LW, editors. Pediatric Neuropsychology Case Studies: From the Exceptional to the Commonplace. New York: Springer Science + Businness Media LLC; 2008.

25. Gibson J, Adams C, Lockton E, Green J. Social communication disorder outside autism? A diagnostic classification approach to delineating pragmatic language impairment, high functioning autism and specific language impairment. J Child Psychol Psychiatry. 2013;54(11): 1186-1197.

26. Bauminger N, Edelsztein HS, Morash J. Social information processing and emotional understanding in children with LD. J Learn Disabil. 2005;38:45-61.

27. Marton I, Wiener J, Rogers M, Moore C, Tannock R. Empathy and social perspective taking in children with attention-deficit/hyperactivity disorder. J Abnorm Child Psychol. 2009;37:107-118.

28. Samuels J, Shugart YY, Wang Y, et al. Clinical correlates and genetic linkage of social and communication difficulties in families with obsessive-compulsive disorder: results from the OCD Collaborative Genetics Study. Am J Med Genet B Neuropsychiatr Genet. 2014; $165 \mathrm{~B}(4): 326-336$.

29. Bloom E, Heath N. Recognition, expression and understanding facial expressions of emotion in adolescents with nonverbal and general learning disabled. J Learn Disabil. 2009;43(2):180-192.
30. Guyer AE, McClure EB, Adler AD, et al. Specificity of facial expression labeling deficits in childhood psychopathology. J Child Psychol Psychiatry. 2007;48(9):863-871.

31. Norbury CF. Practitioner review: social (pragmatic) communication disorder conceptualization, evidence and clinical implications. J Child Psychol Psychiatry. 2014;55(3):204-216.

32. Reisinger LM, Cornish KM, Fombonne E. Diagnostic differentiation of autism spectrum disorders and pragmatic language impairment. J Autism Dev Disord. 2011;41(12):1694-1704.

33. Constantino JN, Gruber CP, Davis S, Hayes S, Passanante N, Przybeck T. The factor structure of autistic traits. J Child Psychol Psychiatry. 2004;45(4):719-726.

34. Hoekstra RA, Bartels M, Cath DC, Boomsma DI. Factor structure, reliability and criterion validity of the Autism Spectrum Quotient (AQ): a study in Dutch population and patient groups. J Autism Dev Disord. 2008;38(8):1555-1566.

35. Frazier TW, Georgiades S, Bishop SL, Hardan AY. Behavioral and cognitive characteristics of females and males with autism in the Simons Simplex Collection. J Am Acad Child Adolesc Psychiatry. 2014;53(3):329-340.

36. Pooni J, Ninteman A, Bryant-Waugh R, Nicholls D, Mandy W. Investigating autism spectrum disorder and autistic traits in early onset eating disorder. Int J Eat Disord. 2012;45(4):583-591.

37. Lord C, Jones RM. Annual research review: re-thinking the classification of autism spectrum disorders. J Child Psychol Psychiatry. 2012;53(5):490-509.

38. American Psychiatric Association. Diagnostic and Statistical Manual of Mental Disorders (DSM-IV) 4th ed. Washington, DC: American Psychiatric Association; 1994

39. Baio J. Prevalence of autism spectrum disorder among children aged 8 years - autism and developmental disabilities monitoring network, 11 sites, United States, 2010. MMWR Surveill Summ. 2014; 63(SS02):1-21.

40. Blumberg SJ, Bramlett MD, Kogan MD, Schieve LA, Jones JR, Lu MC. Changes in prevalence of parent-reported autism spectrum disorder in school-aged U.S. children: 2007 to 2011-2012. Nat Health Statist Rep. 2013;65:1-12.

41. Kim YS, Fombonne E, Koh YJ, Kim SJ, Cheon KA, Leventhal BL. A comparison of DSM-IV pervasive developmental disorder and DSM-5 autism spectrum disorder prevalence in an epidemiologic sample. J Am Acad Child Adolesc Psychiatry. 2014;53(5):500-508.

42. St Pourcain B, Skuse DH, Mandy WP, et al. Variability in the common genetic architecture of social-communication spectrum phenotypes during childhood and adolescence. Mol Autism. 2014;5(1):18.

43. Swineford LB, Thurm A, Baird G, Wetherby AM, Swedo S. Social (pragmatic) communication disorder: a research review of this new DSM-5 diagnostic category. J Neurodev Disord. 2014;6(1):41.

44. Tufan E. The relationship between social communication disorder (SCD) and broad autism phenotype (BAP). J Am Acad Child Adolesc Psychiatry. 2014;53(10):1130.

45. Pruett JR Jr. BAP: not-quite-autism in infants. J Am Acad Child Adolesc Psychiatry. 2014;53:392-394.

46. Nussbaum AM. The DSM-5 diagnostic interview. The Pocket Guide to the DSM-5 Diagnostic Exam. Washington, DC: American Psychiatric Publishing; 2013:57-67.

47. Benner GJ. Language skills of elementary-aged children with emotional and behavioral disorders. Great Plains Res. 2005;15:251-265.

48. Mackie L, Law J. Pragmatic language and the child with emotional/ behavioural difficulties (EBD): a pilot study exploring the interaction between behaviour and communication disability. Int J Lang Commun Disord. 2010;45(4):397-410.

49. Emirbayer M, Mische A. What is agency? Am J Sociol. 1998;103: 962-1023.

50. Weiss JA, Thomson K, Chan L. A systematic literature review of emotion regulation measurement in individuals with autism spectrum disorder. Autism Res. 2014;7:629-648. 
51. Brukner-Wertman Y, Laor N, Golan O. Social (pragmatic) communication disorder and its relation to the autism spectrum: dilemmas arising from the DSM-5 classification. J Autism Dev Disord. 2016;46(8): 2821-2829.

52. Bellani M, Moretti A, Perlini C, Brambilla P. Language disturbances in ADHD. Epidemiol Psychiatr Sci. 2011;20(4):311-315.

53. Demily C, Rossi M, Chesnoy-Servanin G, et al. Complex phenotype with social communication disorder caused by mosaic supernumerary ring chromosome 19p. BMC Med Genet. 2014;15:132.

54. Campbell DB, Warren D, Sutcliffe JS, Lee EB, Levitt P. Association of MET with social and communication phenotypes in individuals with autism spectrum disorder. Am J Med Genet B Neuropsychiatr Genet. 2010;153B(2):438-446.

55. Sungur AÖ, Schwarting RK, Wöhr M. Early communication deficits in the Shank1 knockout mouse model for autism spectrum disorder: developmental aspects and effects of social context. Autism Res. 2016;9(6):696-709.

56. Qiu A, Adler M, Crocetti D, Miller MI, Mostofsky SH. Basal ganglia shapes predict social, communication, and motor dysfunctions in boys with autism spectrum disorder. J Am Acad Child Adolesc Psychiatry. 2010;49(6):539-551.

57. Chien HY, Gau SS, Hsu YC, et al. Altered cortical thickness and tract integrity of the mirror neuron system and associated social communication in autism spectrum disorder. Autism Res. 2015;8(6):694-708.

58. Lo YC, Chen YJ, Hsu YC, Tseng WI, Gau SS. Reduced tract integrity of the model for social communication is a neural substrate of social communication deficits in autism spectrum disorder. J Child Psychol Psychiatry. 2017;58(5):576-585.
59. Mitchell SR, Reiss AL, Tatusko DH, et al. Neuroanatomic alterations and social and communication deficits in monozygotic twins discordant for autism disorder. Am J Psychiatry. 2009;166(8):917-925.

60. Catani M, Bambini V. A model for social communication and language evolution and development (SCALED). Curr Opin Neurobiol. 2014;28C:165-171.

61. Adams C, Gaile J, Lockton E, Freed J. Integrating language, pragmatics, and social intervention in a single-subject case study of a child with a developmental social communication disorder. Lang Speech Hear Serv Sch. 2015;46(4):294-311.

62. Dykstra JR, Boyd BA, Watson LR, Crais ER, Baranek GT. The impact of the advancing social-communication and play (ASAP) intervention on preschoolers with autism spectrum disorder. Autism. 2012; 16(1):27-44.

63. Baribeau DA, Anagnostou E. Social communication is an emerging target for pharmacotherapy in autism spectrum disorder - a review of the literature on potential agents. J Can Acad Child Adolesc Psychiatry. 2014;23(1):20-30.

64. Logan K, Iacono T, Trembath D. A systematic review of research into aided AAC to increase social-communication functions in children with autism spectrum disorder. Augment Altern Commun. 2017; 33(1):51-64.

65. Marraffa C. Social communication in autism spectrum disorder not improved by theory of mind interventions. JPaediatr Child Health. 2016; 52(4):461-463.

66. Bartlett CW, Hou L, Flax JF, et al. A genome-scan for loci shared by autism spectrum disorder and language impairment. Am J Psychiatry. 2014;171:72-81.
Neuropsychiatric Disease and Treatment

\section{Publish your work in this journal}

Neuropsychiatric Disease and Treatment is an international, peerreviewed journal of clinical therapeutics and pharmacology focusing on concise rapid reporting of clinical or pre-clinical studies on a range of neuropsychiatric and neurological disorders. This journal is indexed on PubMed Central, the 'PsycINFO' database and CAS,

\section{Dovepress}

and is the official journal of The International Neuropsychiatric Association (INA). The manuscript management system is completely online and includes a very quick and fair peer-review system, which is all easy to use. Visit http://www.dovepress.com/testimonials.php to read real quotes from published authors. 